\title{
Assessment of the association between red blood cell distribution width and disease activity in patients with systemic vasculitis
}

\author{
JING HONG*, BIN ZHU*, XINTIAN CAI, SHANSHAN LIU, SHASHA LIU, QING ZHU, \\ XIAYIRE AIERKEN, AYIGUZAILI AIHEMAITI, TING WU and NANFANG LI
}

Hypertension Center of People's Hospital of Xinjiang Uygur Autonomous Region, NHC Key Laboratory of Hypertension Clinical Research, Hypertension Institute of Xinjiang, Tianshan, Urumqi, Xinjiang 830001, P.R. China

Received October 2, 2019; Accepted January 28, 2021

DOI: $10.3892 /$ etm.2021.10123

\begin{abstract}
The present study aimed to investigate whether red blood cell distribution width (RDW) could serve as a marker for estimating disease activity in patients with systemic vasculitis (SV). A total of 287 patients with SV and 64 ageand sex-matched healthy controls (HCs) were included in the present study. Biochemical indicators and hematologic parameters were evaluated in patients with SV and the HCs. Disease activity was assessed on the basis of the Birmingham Vasculitis Activity Score (BVAS). RDW was significantly elevated in patients with SV compared with HCs $(\mathrm{P}<0.05)$. A similar result was obtained for the comparison of patients with various disease states, active vs. inactive $(\mathrm{P}<0.05)$. RDW was significantly increased in patients with kidney injury compared with patients without kidney injury $(\mathrm{P}<0.05)$. The correlation analysis indicated that there were positive correlations between RDW and BVAS, erythrocyte sedimentation rate, high-sensitivity $\mathrm{C}$-reactive protein, white blood cells and serum creatinine $(\mathrm{Scr}$; all $\mathrm{P}<0.05)$. In addition, there was a significant negative correlation between RDW and hemoglobin levels $(\mathrm{P}<0.05)$. Multivariate logistic regression analysis indicated that RDW was independently correlated with patients with active SV. The combined diagnosis of RDW and Scr indicated that the sensitivity and specificity were 68.6 and $88.9 \%$, respectively, in terms of assessing disease activity in patients with SV. Therefore, the present study suggested that RDW may serve as a useful index for estimating disease activity and kidney injury in patients with SV. Moreover, the combination
\end{abstract}

Correspondence to: Professor Nanfang Li, Hypertension Center of People's Hospital of Xinjiang Uygur Autonomous Region, NHC Key Laboratory of Hypertension Clinical Research, Hypertension Institute of Xinjiang, 91 Tianchi Road, Tianshan, Urumqi, Xinjiang 830001, P.R. China

E-mail: lnanfang2016@sina.com

*Contributed equally

Key words: systemic vasculitis, red blood cell distribution width, disease activity of RDW and Scr may be more effective than RDW alone when assessing the risk of disease activity in patients with SV.

\section{Introduction}

Systemic vasculitis (SV) is an autoimmune disease involving various types of vessels and its primary characteristics are vascular wall injury and necrosis as a result of inflammation (1). However, the pathogenesis behind SV is not fully understood. Although SV has received increasing attention, as SV can occur in patients of all ages, the clinical manifestations are complex and diverse, and there is a lack of specific markers (2). Therefore, diagnosing and estimating the disease activity of SV is difficult. If left untreated, SV progresses to permanent organ damage, resulting in poor health, poor quality of life, premature death and other manifestations, which ultimately result in a heavy socio-economic burden (3). Therefore, identifying novel markers for estimating the disease activity in patients with SV is important $(4,5)$.

Red blood cell distribution width (RDW), routinely reported as a parameter of the standard automated complete blood count, is a reflection of the variability in the erythrocyte size in the circulation (6). In the past few decades, RDW has been widely used in clinical practice as a tool for analyzing and identifying types of anemia (7). In recent years, the value of RDW has attracted increasing attention and its application scope has become increasingly extensive (8). RDW is considered as an inflammatory related index and research has revealed that it has a potential for predicting the overall mortality in a variety of human inflammatory diseases $(9,10)$. It has also been indicated that increased RDW is associated with autoimmune diseases, such as rheumatoid arthritis and systemic lupus erythematosus (10-13). However, the role of RDW in assessing the disease activity, especially in polyarteritis nodosa (PAN), is not completely understood. Therefore, the present study aimed to investigate whether RDW was increased in patients with SV and could serve as a reliable marker to evaluate disease activity.

\section{Patients and methods}

Study subjects. A total of 287 patients with SV who received diagnosis and treatment at the People's Hospital of Xinjiang 
Uygur Autonomous Region (Urumqi, China) between January 2010 and December 2016 were included as the disease group (Female, $n=147$, Male, $n=140 ;$ Age, 49.02 \pm 16.52 years). Patients were diagnosed with SV according to the 2012 revised International Chapel Hill Consensus Conference classification criteria or the 1990 American College of Rheumatology (14-18). Inclusion criteria for the classification of Churg-Strauss syndrome (CSS) (14): i) Asthma (history of wheezing or diffuse high-pitched rales on expiration); ii) Eosinophilia (Eosinophils $>10 \%$ on white blood cell differential count); iii) mononeuropathy or polyneuropathy [development of mononeuropathy, multiple mononeuropathies or polyneuropathy (i.e. glove/stocking distribution) attributable to a systemic vasculitis]; iv) non-fixed pulmonary infiltrates [migratory or transitory pulmonary non-fixed infiltrates on radiographs (not pulmonary infiltrates, including fixed infiltrates, attributable to systemic vasculitis)]; v) paranasal sinus abnormality (history of acute or chronic paranasal sinus pain or tenderness or radiographic opacification of the paranasal sinuses); and vi) extravascular eosinophils (biopsy, including artery, arteriole or venule, showing accumulations of eosinophils in extravascular areas). For purposes of classification, a patient should have CSS $\geq$ four of these six criteria. Criteria for the classification of Wegener's granulomatosis (WG) (15): i) Nasal or oral inflammation (development of painful or painless oral ulcers, purulent or bloody nasal discharge); ii) abnormal chest radiograph (chest radiograph showing the presence of nodules, fixed infiltrates or cavities); iii) urinary sediment [Microhematuria ( $>5$ red blood cells per high power field) or red cell casts in urine sediment]; iv) granulomatous inflammation on biopsy [histological changes showing granulomatous inflammation within the wall of an artery or in the perivascular or extravascular area (artery or arteriole)]; For purposes of classification, a patient should have WG $\geq$ two of these four criteria are present. There is no uniform standard for the diagnosis of microscopic polyangiitis (MPA), which must be distinguished from PAN and WG before diagnosis. The following conditions contribute to the diagnosis of MPA (16): i) Middle-aged and elderly ( $\geq 45$ years), but more commonly seen in men; ii) renal involvement (proteinuria, hematuria or/and acute renal insufficiency); iii) pulmonary involvement (cough, hemoptysis, dyspnea, pulmonary inflammation and pulmonary renal syndrome); iv) with gastrointestinal (including nausea, vomiting, gastrointestinal bleeding, ischemic abdominal pain, ulcer, intestinal perforation, vascular infarction, intestinal obstruction, melena, hematochezia and peritonitis), heart (including chest pain, heart murmur, palpitation, valve disease, angina pectoris, myocardial infarction, congestive heart failure, hypertension, pericarditis, pericardial effusion and cardiomyopathy), eyes (including exophthalmos, eye pain, optic nerve and eye muscle damage, blurred vision, vision loss, conjunctivitis, corneal ulcer, episcleritis, iritis, retinal vasculitis, visual impairment, ischemic retinopathy or hypertensive retinopathy), ears (including conductive deafness and sensorineural deafness), joints (including joint pain, myalgia, muscle pain, arthritis, intermittent movement disorder of upper and lower limbs) and other organs involved in the performance of the whole body; v) ANCA (indirect fluorescence immunoassay was used for detection) positive; and vi) renal and lung biopsy is helpful in diagnosis for MPA. Criteria for the classification of PAN (17): i) Weight loss $\geq 4 \mathrm{~kg}$ (loss of $\geq 4 \mathrm{~kg}$ body weight since illness began, not due to dieting or other factors); ii) Livedo reticularis (mottled reticular pattern over the skin of portions of the extremities or torso); iii) testicular pain or tenderness (pain or tenderness of the testicles not due to infection, trauma or other causes); iv) myalgias, weakness or leg tenderness [diffuse myalgias (excluding shoulder and hip girdle) or weakness of muscles or tenderness of leg muscles]; v) mononeuropathy or polyneuropathy (development of mononeuropathy, multiple mononeuropathies or polyneuropathy); vi) diastolic blood pressure (BP) $>90 \mathrm{~mm} \mathrm{Hg}$ (development of hypertension with the diastolic BP $>90 \mathrm{~mm} \mathrm{Hg}$ ); vii) Elevated blood urea nitrogen (BUN) creatinine (elevation of BUN $>40 \mathrm{mg} / \mathrm{dl}$ or creatinine $>1.5 \mathrm{mg} / \mathrm{dl}$ not due to dehydration or obstruction); viii) hepatitis B viral infection (Presence of hepatitis B surface antigen or antibody in serum); ix) arteriographic abnormality (arteriogram showing aneurysms or occlusions of the visceral arteries, not due to arteriosclerosis, fibromuscular dysplasia, or other noninflammatory causes); and x) biopsy of the small or medium-sized artery containing PAN (histological changes showing the presence of granulocytes or mononuclear granulocytes). For classification purposes, a patient should have PAN $\geq$ three of these 10 criteria. Criteria for the classification of Takayasu arteritis (TA) (18): i) Age at disease onset $\leq 40$ years (development of symptoms or findings related to TA at age $\leq 40$ years); ii) claudication of extremities (development and worsening of fatigue and discomfort in muscles of $\geq$ one extremity whilst in use, especially the upper extremities); iii) Decreased brachial artery pulse (decreased pulsation of one or both of the brachial arteries); iv) BP difference $>10 \mathrm{~mm} \mathrm{Hg}$ (Difference of $>10 \mathrm{~mm} \mathrm{Hg}$ in systolic blood pressure between arms); v) Bruit over subclavian arteries or aorta (bruit audible on auscultation over one or both of the subclavian arteries or abdominal aorta); vi) arteriogram abnormality (arteriographic narrowing or occlusion of the entire aorta, its primary branches or large arteries in the proximal upper or lower extremities, not due to arteriosclerosis, fibromuscular dysplasia, or similar causes; changes usually focal or segmental). For purposes of classification, a patient would be diagnosed with Takayasu arteritis if $\geq$ three of these six criteria are present. Exclusion criterion: Patients with secondary vasculitis, systemic lupus erythematosus, rheumatoid arthritis, malignancy, infection or with any other coexisting renal disease, including anti-glomerular basement membrane nephritis, IgA nephropathy, diabetic nephropathy or lupus nephritis.

A further 64 age- and sex-matched healthy controls (HC) were included as the control group (female, $n=34$, male, $n=30$; age, $48.13 \pm 11.03$ years). All participants signed informed consent and the study protocol was approved by the Ethics Committee of Xinjiang People's Hospital (Urumqi, China).

Data collection and measurements. All clinical data were obtained from the medical records of patients with SV during hospitalization. The following laboratory parameters were assessed: Hematological parameters, high-sensitivity C-reactive protein (Hs-CRP), serum creatinine (Scr), erythrocyte sedimentation rate (ESR). RDW, white blood cell (WBC), red blood cell (RBC), hemoglobin (HB) and platelet counts were measured by electrical impedance testing method, products of Sysmex diagnostic and using a Sysmex XN 9000 
Table I. Demographic and clinical characteristics in systemic vasculitis patients and healthy controls.

\begin{tabular}{lccccccr}
\hline Parameters & SV, $\mathrm{n}=287$ & $\mathrm{HC}, \mathrm{n}=64$ & P-value $^{\mathrm{c}}$ & AAV $(\mathrm{n}=170)$ & PAN $(\mathrm{n}=73)$ & TA $(\mathrm{n}=44)$ & P-value $^{\mathrm{d}}$ \\
\hline Age, years & $49.02 \pm 16.52$ & $48.13 \pm 11.03$ & 0.597 & $56.29 \pm 15.52^{\mathrm{a}}$ & $41.36 \pm 10.89^{\mathrm{a}, \mathrm{b}}$ & $34.00 \pm 11.85$ & $<0.001$ \\
Female, $\mathrm{n}(\%)$ & $147(51.2)$ & $34(53.1)$ & 0.783 & $82(48.2)^{\mathrm{a}}$ & $34(46.6)^{\mathrm{a}}$ & $31(70.5)$ & 0.021 \\
WBC, $\mathrm{x} 10^{9} / \mathrm{l}$ & $8.36 \pm 3.21$ & $6.25 \pm 1.43$ & $<0.001$ & $8.90 \pm 3.48$ & $7.31 \pm 2.21^{\mathrm{b}}$ & $7.95 \pm 3.04$ & 0.001 \\
$\mathrm{RBC}, \mathrm{x} 10^{9} / 1$ & $4.21 \pm 0.88$ & $4.78 \pm 0.50$ & $<0.001$ & $3.87 \pm 0.93^{\mathrm{a}}$ & $4.73 \pm 0.53^{\mathrm{b}}$ & $4.67 \pm 0.65$ & $<0.001$ \\
HB, g/l & $122.13 \pm 27.88$ & $144.73 \pm 12.45$ & $<0.001$ & $113.40 \pm 28.67^{\mathrm{a}}$ & $138.31 \pm 18.41^{\mathrm{b}}$ & $132.79 \pm 14.56$ & $<0.001$ \\
PLT, x 10 $/ 1$ & $238.23 \pm 89.56$ & $251.33 \pm 66.80$ & 0.189 & $231.29 \pm 91.02$ & $237.10 \pm 69.54$ & $262.45 \pm 105.88$ & 0.154 \\
RDW, \% & $14.13 \pm 1.73$ & $12.67 \pm 0.66$ & $<0.001$ & $14.50 \pm 1.82^{\mathrm{a}}$ & $13.52 \pm 1.57^{\mathrm{b}}$ & $13.87 \pm 1.26$ & $<0.001$ \\
ESR, mm/h & $31.52 \pm 25.49$ & $11.13 \pm 8.11$ & $<0.001$ & $39.27 \pm 27.79^{\mathrm{a}}$ & $18.60 \pm 11.89^{\mathrm{b}}$ & $25.63 \pm 23.55$ & $<0.001$ \\
Hs-CRP, mg/l & $23.76 \pm 46.10$ & $1.82 \pm 2.61$ & $<0.001$ & $36.41 \pm 56.22^{\mathrm{a}}$ & $4.31 \pm 5.91^{\mathrm{b}}$ & $8.13 \pm 14.18$ & $<0.001$ \\
Scr, mg/dl & $204.28 \pm 240.26$ & $63.50 \pm 17.27$ & $<0.001$ & $276.96 \pm 287.90^{\mathrm{a}}$ & $100.55 \pm 43.87^{\mathrm{b}}$ & $91.62 \pm 41.90$ & $<0.001$ \\
BVAS & $9.54 \pm 6.68$ & - & - & $10.82 \pm 6.88^{\mathrm{a}}$ & $8.11 \pm 5.67^{\mathrm{b}}$ & $7.00 \pm 6.34$ & $<0.001$ \\
\hline
\end{tabular}

Data are presented as the mean \pm SD or as the count (percentage). SV vs. HC: Age, WBC, RBC, HB, PLT and RDW were compared using the independent sample Student's t-test. The female \% was compared using the $\chi^{2}$ test. ESR, Hs-CRP and Scr were compared using the Kruskal-Wallis test. 'Independent sample Student's t-test, $\chi^{2}$ test or Kruskal Wallis test. AAV vs. PAN vs. TA: Age, WBC, RBC, HB, PLT and RDW were compared using one-way ANOVAs and post hoc Student-Newman-Keuls. The female $\%$ was compared using the $\chi^{2}$ test. ESR, Hs-CRP, Scr and BVAS were compared using the Kruskal-Wallis tests and post hoc Dunn's tests. ${ }^{\mathrm{a}} \mathrm{P}<0.05$ vs. TA; ${ }^{\mathrm{b}} \mathrm{P}<0.05$ vs. AAV. ${ }^{\mathrm{d} O n e-w a y ~ A N O V A, ~} \chi^{2}$ test or Kruskal Wallis test. AAV, anti-neutrophil cytoplasmic antibody associated vasculitis; BVAS, Birmingham Vasculitis Activity Score; ESR, erythrocyte sedimentation rate; HB, hemoglobin; HC, healthy controls; Hs-CRP, high-sensitivity C-reactive protein; PAN, polyarteritis nodosa; PLT, platelets; RBC, red blood cell; RDW, red blood cell distribution width; Scr, serum creatinine; SV, systemic vasculitis; TA, takayasu arteritis; WBC, white blood cell.

Hematology analyzer (Sysmex GmbH). ESR was measured by using a Model Minitor-100 (light emitting diodes and photocells were used to detect the change of light transmittance at the interface between red blood cells and plasma, where the ESR value was obtained). Hs-CRP was performed using a turbid metric method and diagnostic products of Olympus diagnostic, using an Olympus AU-2700 Chemistry Analyzer (Olympus Corporation). Scr was measured by enzyme colorimetry method, products of Olympus diagnostic and using an Olympus AU-2700 Chemistry Analyzer (Olympus Corporation).

Definitions of disease activity. Disease activity was measured using the third version of the Birmingham Vasculitis Activity Score (BVAS) (19). Active disease was defined as a BVAS $\geq 1$, whereas inactive disease was defined as BVAS $=0$.

Definitions of kidney injury. The presence of increased Scr, proteinuria and/or hematuria was taken to indicate kidney injury. Increased Scr was defined as Scr $>84 \mu \mathrm{mol} / 1$ or $>104 \mu \mathrm{mol} / 1$ for female and male patients, respectively. Proteinuria was defined as $>1+$ in the routine urine collection or in a 24 -h urine collection, containing $\geq 150 \mathrm{mg}$ protein. Hematuria was defined as $\geq$ five red blood cells per high-power field (light microscopy; magnification, x400; Olympus Corporation) in urine sediment $(20,21)$.

Statistical analysis. Statistical analyses were performed using SPSS software (version 20.0; IBM Corp). Continuous variables are presented as the mean \pm SD or median (interquartile range). Categorical variables are presented as the number $(\%)$. Comparisons among groups were analyzed using an independent sample Student's t-test or one-way ANOVA. Multiple comparison tests were performed using the Student-Newman-Keuls test. The Kruskal-Wallis test was used to test data that was not normally distributed. Multiple comparison tests were performed using the Dunn's post hoc test. Categorical variables were evaluated using the $\chi^{2}$ test. Binary logistic regression was used to identify independent factors of disease activity in patients with SV. Correlation between numerical data was calculated using Spearman's or Pearson's correlation coefficient. A receiver-operating characteristic (ROC) curve was used to determine a cut-off value with the highest sensibility and specificity for estimating the disease activity in patients with SV. In addition, to further improve sensitivity or specificity, multiple biomarkers were used for combined diagnosis, and binary logistic regression analysis and ROC curves were established (22). $\mathrm{P}<0.05$ was considered to indicate a statistically significant difference.

\section{Results}

Characteristics of patients with SV and HC. A total of 287 patients with SV and $64 \mathrm{HCs}$ were recruited. Among the patients with SV, 170 were diagnosed as anti-neutrophil cytoplasmic antibody associated vasculitis (AAV), 73 were diagnosed with PAN and 44 were diagnosed with TA) Among them, 193 patients had active SV and 94 had inactive SV. Moreover, 149 patients displayed kidney injury and 138 patients did not display kidney injury. Demographic and clinical characteristics of patients and HCs are presented in Tables I and II. There were no significant differences in the age and sex distribution of patients with $\mathrm{SV}$ and $\mathrm{HCs}$ (all $\mathrm{P}>0.05$ ).

$R D W$ in patients with $S V$ and $H C$. RDW in the SV group was significantly increased compared with matched HCs $(14.13 \pm 1.73$ vs. $12.67 \pm 0.66$; $\mathrm{P}<0.05$; Fig. 1A). RDW was higher in the active stage group compared with the inactive 
Table II. Demographic and clinical characteristics of patients with active stage, inactive stage and healthy controls.

\begin{tabular}{|c|c|c|c|c|}
\hline Parameters & Active, $n=193$ & Inactive, $\mathrm{n}=94$ & $\mathrm{HC}, \mathrm{n}=64$ & P-value ${ }^{c}$ \\
\hline Age, years & $51.91 \pm 16.31$ & $43.15 \pm 15.57^{\mathrm{a}, \mathrm{b}}$ & $48.13 \pm 11.03$ & $<0.001$ \\
\hline Female, n (\%) & $92(47.7)$ & $55(58.5)$ & $34(53.1)$ & 0.217 \\
\hline $\mathrm{WBC}, \times 10^{9} / 1$ & $8.79 \pm 3.32^{b}$ & $7.45 \pm 2.76^{\mathrm{a}, \mathrm{b}}$ & $6.25 \pm 1.43^{\mathrm{a}}$ & $<0.001$ \\
\hline $\mathrm{RBC}, \mathrm{x} 10^{9} / 1$ & $4.06 \pm 0.96^{\mathrm{b}}$ & $4.50 \pm 0.61^{\mathrm{a}, \mathrm{b}}$ & $4.78 \pm 0.50^{\mathrm{a}}$ & $<0.001$ \\
\hline $\mathrm{HB}, \mathrm{g} / \mathrm{l}$ & $117.01 \pm 29.56^{\mathrm{b}}$ & $132.93 \pm 20.17^{\mathrm{a}, \mathrm{b}}$ & $144.73 \pm 12.45^{\mathrm{a}}$ & $<0.001$ \\
\hline PLT, $x 10^{9} / 1$ & $235.44 \pm 93.82$ & $244.03 \pm 80.18$ & $251.33 \pm 66.80$ & 0.402 \\
\hline RDW, \% & $14.45 \pm 1.82^{\mathrm{b}}$ & $13.48 \pm 1.30^{\mathrm{a}, \mathrm{b}}$ & $12.67 \pm 0.66^{\mathrm{a}}$ & $<0.001$ \\
\hline $\mathrm{ESR}, \mathrm{mm} / \mathrm{h}$ & $36.53 \pm 26.69^{b}$ & $20.83 \pm 18.78^{\mathrm{a}, \mathrm{b}}$ & $11.13 \pm 8.11^{\mathrm{a}}$ & $<0.001$ \\
\hline Hs-CRP, mg/l & $28.85 \pm 50.59^{\mathrm{b}}$ & $13.52 \pm 33.35^{\mathrm{a}, \mathrm{b}}$ & $1.82 \pm 2.61^{\mathrm{a}}$ & $<0.001$ \\
\hline Scr, mg/dl & $265.21 \pm 271.04^{\mathrm{b}}$ & $76.39 \pm 31.12^{\mathrm{a}}$ & $63.50 \pm 17.27^{\mathrm{a}}$ & $<0.001$ \\
\hline
\end{tabular}

Data are presented as mean \pm SD or as the count (percentage). Age, WBC, RBC, HB, PLT and RDW were compared using one-way ANOVAs with post hoc Student-Newman-Keuls tests. The female $\%$ was compared using the $\chi^{2}$ test. ESR, Hs-CRP and Scr were compared using

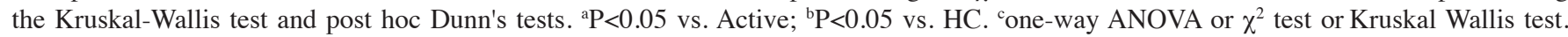
ESR, erythrocyte sedimentation rate; HB, hemoglobin; Hs-CRP, high-sensitivity C-reactive protein; PLT, platelets; RBC, red blood cell; RDW, red blood cell distribution width; Scr, serum creatinine; WBC, white blood cell.
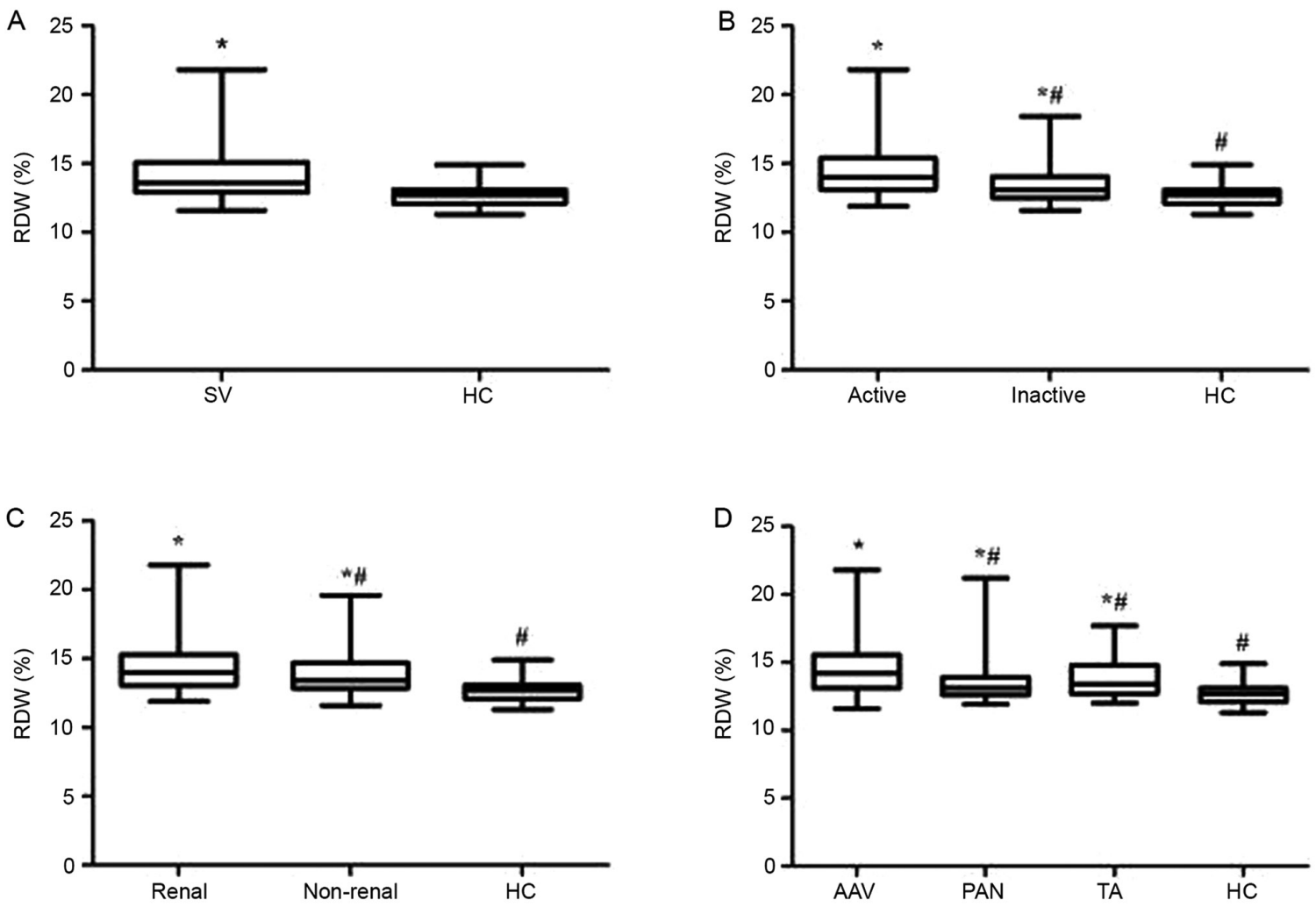

Figure 1. RDW in the various groups. (A) RDW in patients with SV and HCs. "P<0.05 vs. HC. (B) RDW in patients with active and inactive SV. "P<0.05 vs. HC; ${ }^{\text {"}} \mathrm{P}<0.05$ vs. active. (C) RDW in patients with SV with kidney injury and non-kidney injury. ${ }^{*} \mathrm{P}<0.05$ vs. HC; ${ }^{*} \mathrm{P}<0.05$ vs. kidney injury. (D) RDW in SV subsets. ${ }^{\text {"P}}<0.05$ vs. HC; " $\mathrm{P}<0.05$ vs. AAV. AAV, antineutrophil cytoplasmic antibody associated vasculitis; HC, healthy control; PAN, polyarteritis nodosa; RDW, red blood cell distribution width; SV, systemic vasculitis; TA, takayasu arteritis.

stage group (14.45 \pm 1.82 vs. $13.48 \pm 1.30 ; \mathrm{P}<0.05$; Fig. $1 \mathrm{~B})$. In addition, the RDW was significantly different between patients with kidney injury and patients without kidney injury $(14.35 \pm 1.84$ vs. $13.89 \pm 1.56$; $\mathrm{P}<0.05$; Fig. $1 C)$. In the subgroup analysis, the RDW of each subgroup, including TA, PAN and AAV, was higher compared with the HC group (AAV vs. HC,
$14.50 \pm 1.82$ vs. $12.67 \pm 0.66, \mathrm{P}<0.05 ; \mathrm{PAN}$ vs. $\mathrm{HC}, 13.52 \pm 1.57$ vs $12.67 \pm 0.66, \mathrm{P}<0.05$; TA vs. HC, $13.87 \pm 1.26$ vs. $12.67 \pm 0.66$, $\mathrm{P}<0.05)$. Moreover, RDW was increased in patients with AAV compared with patients with PAN or TA (AAV vs. $\mathrm{PAN}, 14.50 \pm 1.82$ vs. $13.52 \pm 1.57, \mathrm{P}<0.05$; AAV vs. TA, $14.50 \pm 1.82$ vs. $13.87 \pm 1.26, \mathrm{P}<0.05$; Fig. $1 \mathrm{D})$. RDW was not 

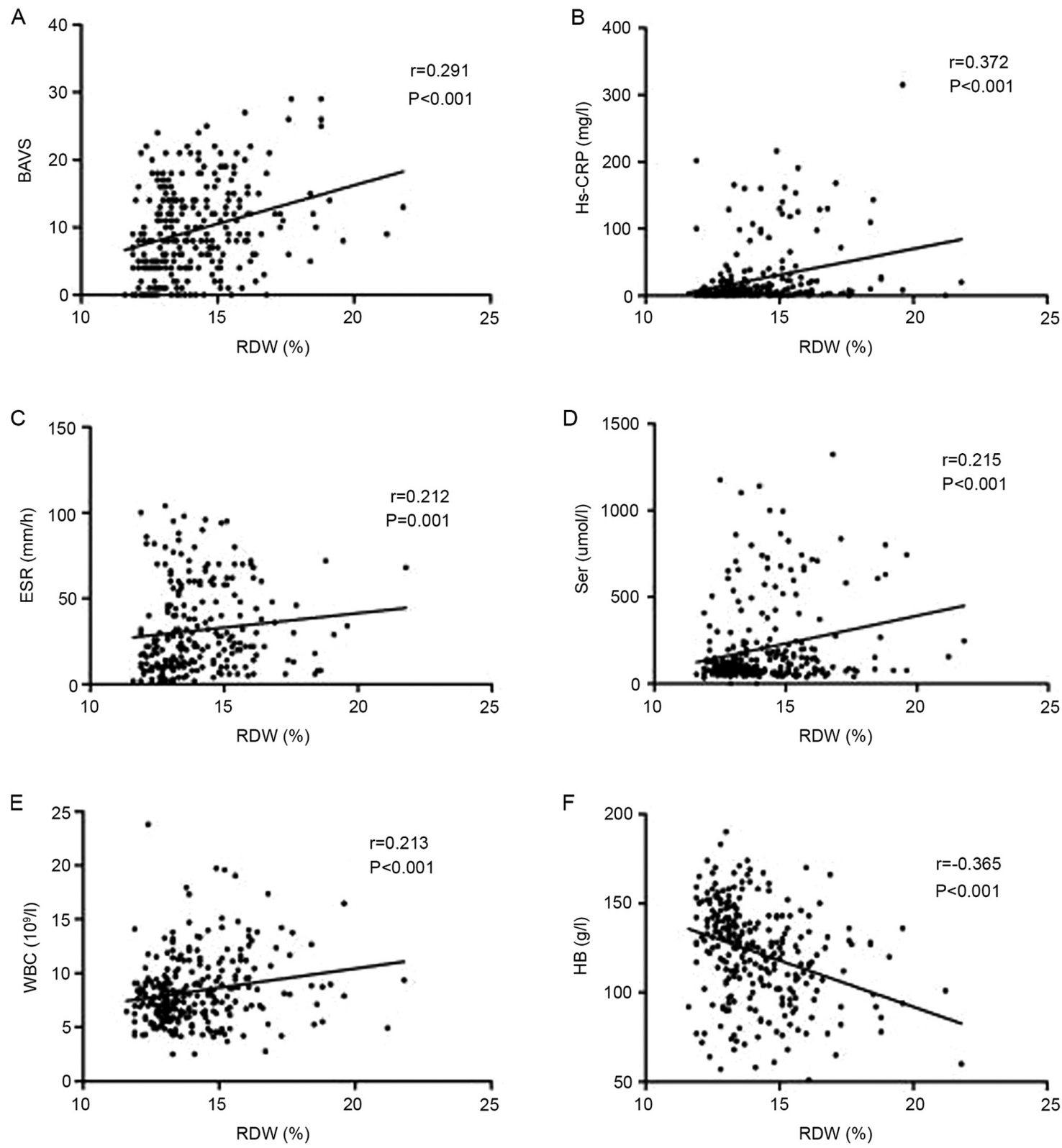

Figure 2. Correlations of RDW with BVAS, Hs-CRP, ESR, Scr, WBC and HB. The correlations between RDW and (A) BVAS, (B) Hs-CRP, (C) ESR, (D) Scr, (E) WBC and (F) HB were analyzed in patients with systemic vasculitis. BVAS, Birmingham Vasculitis Activity Score; ESR, erythrocyte sedimentation rate; HB, hemoglobin; Hs-CRP, high-sensitivity C-reactive protein; RDW, red blood cell distribution width; Scr, serum creatinine; WBC, white blood cell.

significantly different between the patients with PAN and patients with TA (13.52 \pm 1.57 vs. $13.87 \pm 1.26$; P>0.05; Fig. 1D).

Correlation between $R D W$ and other parameters in patients with $S V$. The relationship between RDW and laboratory parameters in patients with SV was assessed. The results indicated that there was positive correlation between RDW and BVAS $(\mathrm{r}=0.291 ; \mathrm{P}<0.001)$, Hs-CRP $(\mathrm{r}=0.372 ; \mathrm{P}<0.001)$, ESR $(r=0.212 ; P=0.001)$, Scr $(r=0.215 ; P<0.001)$ and $\mathrm{WBC}$ $(\mathrm{r}=0.213 ; \mathrm{P}<0.001)$ (Fig. 2A-E). By contrast, the opposite result was obtained between RDW and HB ( $r=-0.365$; $\mathrm{P}<0.001$; Fig. 2F). Multivariate logistic regression analysis suggested that RDW [odds ratio $(\mathrm{OR})=1.500 ; 95 \%$ confidence interval $(\mathrm{CI})=1.101-2.042 ; \mathrm{P}=0.010]$ and $\mathrm{Scr}[\mathrm{OR}=1.024$; 95\% $\mathrm{CI}=1.013-1.045 ; \mathrm{P}<0.001]$ were independently associated with patients with active SV (Table III).
ROC curve analysis to identify optimal cutoff values of RDW. When assessing the presence of disease activity with RDW in patients with SV, a cut-off value of 13.65 , with $57.3 \%$ sensitivity and $67.0 \%$ specificity was observed according to ROC curve analysis [area under the curve (AUC) $=0.68 ; 95 \% \mathrm{CI}=0.61-0.74$; Fig. 3A]. Furthermore, binary logistic regression and ROC curves assessed the combined diagnostic efficiency of multiple parameters. The combination of RDW and Scr, after adjusting by the regression coefficient of the binary logistic regression, had $68.6 \%$ sensitivity and $88.9 \%$ specificity for diagnosing patients with active SV (AUC=0.84; 95\% CI=0.80-0.89; Fig. 3B).

\section{Discussion}

The extent of disease activity or relapse serves an important role in the early individualized therapy and prognostic 
Table III. Multivariate logistic regression analysis of patients with active stage versus inactive stage.

\begin{tabular}{|c|c|c|c|c|c|c|}
\hline \multirow[b]{2}{*}{ Variable } & \multicolumn{3}{|c|}{ Univariate analysis } & \multicolumn{3}{|c|}{ Multivariate analysis } \\
\hline & OR & $95 \% \mathrm{CI}$ & P-value & OR & $95 \% \mathrm{CI}$ & P-value \\
\hline Age & 1.035 & $1.018-1.052$ & $<0.001$ & 0.984 & $0.957-1.012$ & 0.261 \\
\hline Female & 0.639 & $0.388-1.052$ & 0.078 & 0.599 & $0.255-1.406$ & 0.239 \\
\hline WBC & 1.167 & $1.062-1.283$ & 0.001 & 1.136 & $0.959-1.346$ & 0.140 \\
\hline HB & 0.977 & $0.966-0.987$ & $<0.001$ & 1.009 & $0.986-1.033$ & 0.435 \\
\hline PLT & 0.999 & $0.996-1.002$ & $<0.001$ & 0.998 & 0.993-1.004 & 0.536 \\
\hline RDW & 1.533 & $1.260-1.865$ & $<0.001$ & 1.500 & $1.101-2.042$ & 0.010 \\
\hline ESR & 1.031 & $1.017-1.046$ & $<0.001$ & 1.018 & $0.991-1.045$ & 0.190 \\
\hline Hs-CRP & 1.010 & $1.002-1.018$ & 0.018 & 0.994 & 0.979-1.009 & 0.413 \\
\hline Scr & 1.022 & $1.014-1.031$ & $<0.001$ & 1.024 & $1.013-1.045$ & $<0.001$ \\
\hline
\end{tabular}

CI, confidence interval; ESR, erythrocyte sedimentation rate; HB, hemoglobin; Hs-CRP, high-sensitivity C-reactive protein; OR, odds ratio; PLT, platelets; RDW, red blood cell distribution width; Scr, serum creatinine; WBC, white blood cell.
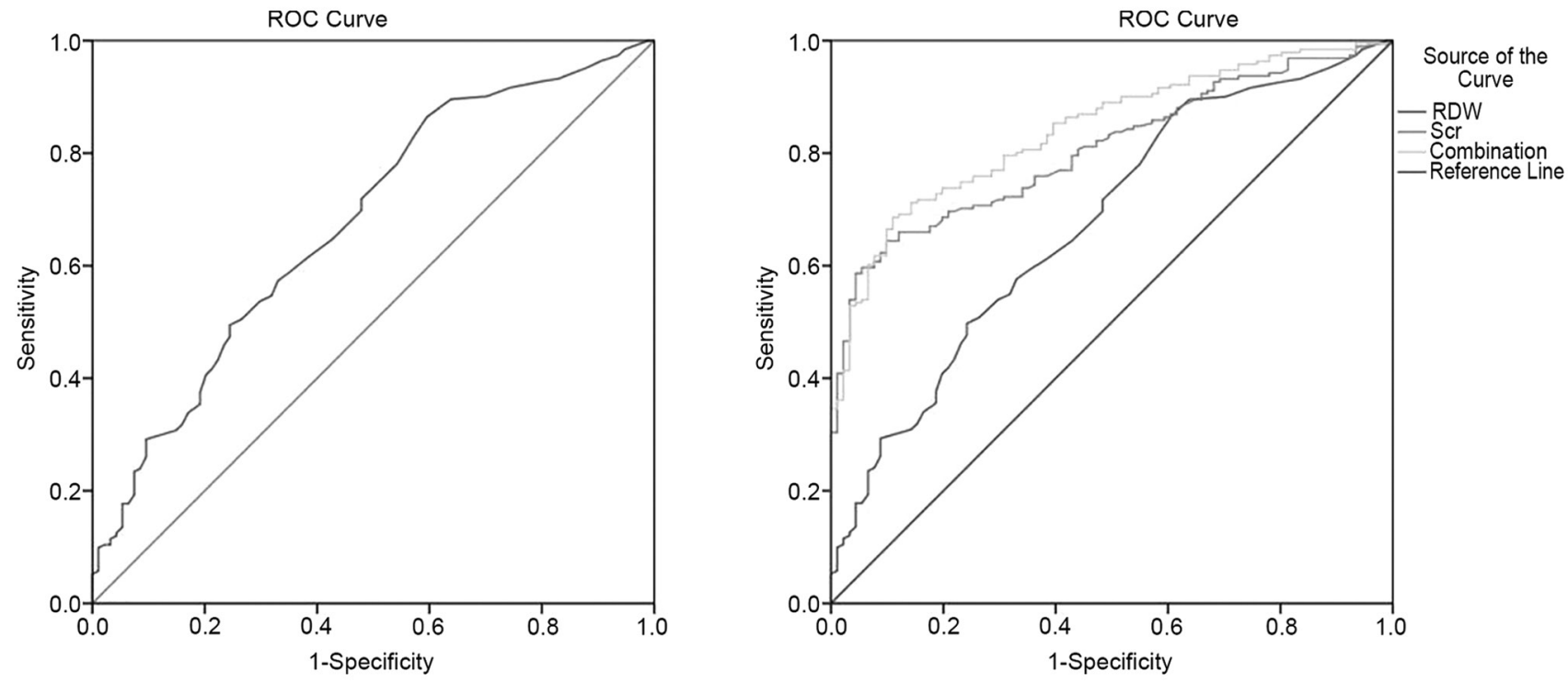

Figure 3. ROC curves in patients with SV. ROC curve of RDW for identifying patients with active SV. ROC curve of the combination of the RDW and Scr for identifying patients with active SV. RDW, red blood cell distribution width; ROC, receiver operating characteristic; Scr, serum creatinine; SV, systemic vasculitis.

assessment of SV (23). However, the assessment of disease activity and relapses in patients with SV is difficult (24). Although angiography and biopsies are used to assess disease activity and relapses in patients with SV, both strategies display imperfect sensitivity due to sampling error and often contain inconclusive findings (25). Furthermore, the strategies are expensive, invasive, clinically risky and not easily tolerated by all patients (26). Therefore, improved tests and useful markers are required to assess disease activity and relapses in patients with SV. RDW is an indicator of inflammation that has feasibility as a prognostic parameter in various diseases (7). In the clinic, RDW is easily obtained as part of a routine blood examination and provides valuable information in a number of diseases without adding additional financial burden on the patients (8). Recent studies have discovered a relationship between subsets of $\mathrm{SV}$ and RDW (27,28); therefore, the present study investigated whether RDW could serve a role as a marker for estimating the disease activity of SV.

The results of the present study suggested that RDW was significantly elevated in patients with active SV compared with patients with inactive SV or HCs. The correlation analysis indicated that there was positive correlation between RDW and BVAS, Hs-CRP, ESR and WBC. As the P-value was low and the correlation coefficient was $<0.4$, it is unlikely that there was a strong correlation. A potential explanation for this is that the present study was retrospective; therefore, further longitudinal studies are required to verify the results of the present study. In addition, RDW was found to be an independent factor for patients with active SV, which was indicated by multivariate logistic regression analysis. The results suggested that RDW could be a reflection of disease activity in patients with SV. As aforementioned, there was a connection between RDW and SV subsets. RDW has been reported to be 
a potential marker to evaluate disease activity in other forms of vasculitis, including Behçet disease, TA and granulomatosis with polyangiitis (29-31), which was consistent with the results of the present study. In addition, RDW in patients with inactive SV was raised compared with the HCs. The results indicated a potential persistent state of hidden inflammation or tissue/vascular wall injury, but further investigation is required to verify this finding.

Several studies have assessed renal function and extent of renal damage with RDW in various diseases (32-34). The present study investigated whether RDW was related to kidney injury in SV. The results indicated that RDW was elevated in patients with SV with kidney injury compared with patients with SV without kidney injury. The correlation analysis suggested that there was a positive correlation between RDW and Scr, as well as a negative correlation between RDW and HB. Zhu et al (27) indicated that RDW was increased in patients with TA with anemia compared with patients with TA without anemia and control subjects, which was consistent with the present study. However, Kim et al (30) indicated that RDW was not significantly associated with $\mathrm{HB}$, which may be related to the sex and age variations between the enrolled cohorts.

Subgroup analysis of the type of SV was also performed in the present study. The results indicated that RDW was significantly higher in patients with AAV, PAN and TA compared with HCs, which suggested that RDW may participate in the pathogenesis behind SV subsets. However, the exact role of RDW requires further investigation. Additionally, RDW was higher in patients with AAV compared with patients with PAN and TA. A potential explanation may be due to the relatively higher disease activity of patients with AAV. To further assess the disease activity in patients with SV, ROC analysis was conducted. The results suggested that the optimal cut-off value of RDW was 13.65 with $57.3 \%$ sensitivity and $67.0 \%$ specificity. To improve the accuracy and efficiency of diagnosis, identification of patients with active SV is important. Using RDW and Scr as combinatorial markers to construct ROC curves using binary logistic regression indicated that RDW combined with Scr had an advantage compared with RDW alone, with $68.6 \%$ sensitivity and $88.9 \%$ specificity. Therefore, the combination achieved higher values for assessing patients with active SV.

At present, the exact pathogenesis behind the relationship between RDW and SV is not completely understood. A previous study indicated that RDW levels might be affected by inflammatory cytokines, such as IL-1, IL-6 and TNF- $\alpha$ (12). Inflammatory cytokines may affect the function of bone marrow, inhibit the maturation of erythrocytes and increase the RDW (35). Therefore, higher RDW levels may reflect the underlying inflammatory state caused by chronic inflammation, thereby transforming the intracellular homeostasis of red blood cells and impairing the maturation of red blood cells (36). Collectively, the aforementioned findings suggested that cytokines and RDW serve an important role in the pathogenesis of SV.

The results of the present study suggested that RDW may serve as a suitable biomarker for assessing the disease activity of patients with SV and its subgroups. However, the present study had a number of limitations. Firstly, due to the limitations of cross-sectional research, the present study was unable to derive the causal relationship between RDW and $\mathrm{SV}$. Therefore, prospective studies are required to investigate whether RDW predicts the prognosis of SV. Secondly, the present study included patients with anemia, but serum iron, vitamin B12 and folic acid levels were not recorded. Therefore, some of the patients may have had anemia due to vitamin B12 and mineral deficiencies, thus affecting RDW levels. Finally, the relationship between RDW and other sensitive inflammatory markers, such as TNF- $\alpha$, IL-1 and IL-6, was not evaluated.

In conclusion, the results of the present study suggested that RDW might serve as a potential marker for disease activity and kidney injury in patients with SV. Moreover, the combination of RDW and Scr may have a higher value when assessing the risk of disease activity in patients with SV. However, further studies are required to investigate the exact role of RDW in the pathogenesis of SV.

\section{Acknowledgements}

Not applicable.

\section{Funding}

This study was supported by the National Natural Science Foundation of Xinjiang (grant no. 2018D01C117).

\section{Availability of data and materials}

All data generated and/or analyzed during this study are included in this published article.

\section{Authors' contributions}

$\mathrm{JH}$ and BZ analyzed the data and wrote the manuscript. XC, ShanL and ShasL performed the data collection from medical records and participated in the data analysis. QZ, XC and TW participated in the study design and revised the manuscript. $\mathrm{XC}, \mathrm{TW}, \mathrm{XA}$ and AA performed the data collection from medical records. XC and TW revised the manuscript critically for important intellectual content. QZ and TW confirm the authenticity of all the raw data. NL conceived and helped design the study, revised the manuscript and approved the final version of the manuscript. JH and BZ contributed equally to this study, and should be regarded as co-first authors. All authors reviewed and approved the final manuscript.

\section{Ethics approval and consent to participate}

All participants have signed an informed consent form the study protocol was approved by the ethics committee of Xinjiang People's Hospital (Urumqi, China).

\section{Patient consent for publication}

Not applicable.

\section{Competing interests}

The authors declare that they have no competing interests. 


\section{References}

1. Elefante E, Bond M, Monti S, Lepri G, Cavallaro E, Felicetti M Calabresi E, Posarelli C, Talarico R, Quartuccio L and Baldini C: One year in review 2018: Systemic vasculitis. Clin Exp Rheumatol 36 (Suppl 111): S12-S32, 2018.

2. Perez-Alamino R and Maldonado-Ficco H: New insights on biomarkers in systemic vasculitis. Curr Rheumatol Rep 17: 12, 2015

3. Trieste L, Palla I, Baldini C, Talarico R, D'Angiolella L, Mosca M and Turchetti G: Systemic vasculitis: How little we know about their societal and economic burden. Clin Exp Rheumatol 30 (Suppl 73): S154-S156, 2012.

4. Benarous L, Terrier B, Laborde-Casterot H, Bérezné A, Dunogué B, Cohen P, Puéchal X, Mouthon L, Bensefa-Colas L and Guillevin L; French Vasculitis Study Group (FVSG) Employment, work disability and quality of life in patients with ANCA-associated vasculitides. The EXPOVAS study. Clin Exp Rheumatol 35 (Suppl 103): S40-S46, 2017.

5. Barra LJ, Bateman EA, Rohekar S, Pagnoux C and Moradizadeh M: Assessment of work limitations and disability in systemic vasculitis. Clin Exp Rheumatol 34 (Suppl 97): S111-S114, 2016.

6. Yousefi B, Sanaie S, Ghamari AA, Soleimanpour H, Karimian A and Mahmoodpoor A: Red cell distribution width as a novel prognostic marker in multiple clinical studies. Indian J Crit Care Med 24: 49-54, 2020

7. Salvagno GL, Sanchis-Gomar F, Picanza A and Lippi G: Red blood cell distribution width: A simple parameter with multiple clinical applications. Crit Rev Clin Lab Sci 52: 86-105, 2015.

8. Lippi G, Mattiuzzi C and Cervellin G: Learning more and spending less with neglected laboratory parameters: The paradigmatic case of red blood cell distribution width. Acta Biomed 87: 323-328, 2016

9. Hu Z, Sun Y, Wang Q, Han Z, Huang Y, Liu X, Ding C, Hu C, Qin Q and Deng A: Red blood cell distribution width is a potential prognostic index for liver disease. Clin Chem Lab Med 51: 1403-1408, 2013

10. Patel KV,Ferrucci L, Ershler WB, Longo DL and Guralnik JM: Red blood cell distribution width and the risk of death in middle-aged and older adults. Arch Intern Med 169: 515-523, 2009.

11. Hu ZD, Chen Y, Zhang L, Sun Y, Huang YL, Wang QQ, Xu YL, Chen SX, Qin Q and Deng AM: Red blood cell distribution width is a potential index to assess the disease activity of systemic lupus erythematosus. Clin Chim Acta 425: 202-205, 2013.

12. He Y, Liu C, Zeng Z, Ye W, Lin J and Ou Q: Red blood cell distribution width: A potential laboratory parameter for monitoring inflammation in rheumatoid arthritis. Clin Rheumatol 37: $161-167,2018$.

13. Xu H, Fu S, Wang W, Zhang Q, Hu J, Gao L, Zhu W and Gong F: Predictive value of red blood cell distribution width for coronary artery lesions in patients with Kawasaki disease. Cardiol Young 26: 1151-1157, 2016.

14. Masi AT, Hunder GG, Lie JT, Michel BA, Bloch DA, Arend WP, Calabrese LH, Edworthy SM, Fauci AS, Leavitt RY, et al: The American college of rheumatology 1990 criteria for the classification of Churg-Strauss syndrome (allergic granulomatosis and angiitis). Arthritis Rheum 33: 1094-1100, 1990.

15. Leavitt RY, Fauci AS, Bloch DA, Michel BA, Hunder GG, Arend WP, Calabrese LH, Fries JF, Lie JT, Lightfoot RW Jr, et al: The American college of rheumatology 1990 criteria for the classification of Wegener's granulomatosis. Arthritis Rheum 33: 1101-1107, 1990.

16. Jennette JC, Falk RJ, Bacon PA, Basu N, Cid MC, Ferrario F, Flores-Suarez LF, Gross WL, Guillevin L, Hagen EC, et al: 2012 Revised international chapel hill consensus conference nomenclature of vasculitides. Arthritis Rheum 65: 1-11, 2013.

17. Lightfoot RW Jr, Michel BA, Bloch DA, Hunder GG, Zvaifler NJ, McShane DJ, Arend WP, Calabrese LH, Leavitt RY, Lie JT, et al: The American college of rheumatology 1990 criteria for the classification of polyarteritis nodosa. Arthritis Rheum 33: 1088-1093, 1990.
18. Arend WP, Michel BA, Bloch DA, Hunder GG, Calabrese LH, Edworthy SM, Fauci AS, Leavitt RY, Lie JT, Lightfoot RW Jr, et al: The American college of rheumatology 1990 criteria for the classification of Takayasu arteritis. Arthritis Rheum 33: 1129-1134, 1990.

19. Mukhtyar C, Lee R, Brown D, Carruthers D, Dasgupta B, Dubey S, Flossmann O, Hall C, Hollywood J, Jayne D, et al: Modification and validation of the Birmingham vasculitis activity score (version 3). Ann Rheum Dis 68: 1827-1832, 2009.

20. Li N, Zhu B, Zhu Q, Heizati M, Wu T, Wang G, Yao X, Luo Q, Liu S and Liu S: Serum lysosomal-associated membrane protein-2 levels are increased in small and medium-vessel vasculitis, especially in polyarteritis nodosa. Clin Exp Rheumatol 37 (Suppl 117): S79-S85, 2019.

21. Liu S, Li N, Zhu Q, Zhu B, Wu T, Wang G, Liu S and Luo Q Increased serum MCP-1 levels in systemic vasculitis patients with renal involvement. J Interferon Cytokine Res 38: 406-412, 2018.

22. Zhu B, Li N, Zhu Q, Wu T, Heizati M, Wang G, Yao X, Luo Q, Liu S, Liu S and Hong J: Association of serum high mobility group box 1 levels with disease activity and renal involvement in patients with systemic vasculitis. Medicine (Baltimore) 98: e14493, 2019.

23. Eleftheriou D and Brogan PA: Therapeutic advances in the treatment of vasculitis. Pediatr Rheumatol Online J 14: 26, 2016

24. Katsuyama T, Sada KE and Makino H: Current concept and epidemiology of systemic vasculitides. Allergol Int 63: 505-513, 2014.

25. Hatemi G, Esatoglu SN and Yazici Y: Biomarkers in vasculitis. Curr Opin Rheumatol 30: 30-35, 2018.

26. Langford CA: Vasculitis. J Allergy Clin Immunol 125 (Suppl 2): S216-S225, 2010.

27. Zhu X, Zhang M, Lan F, Wei H, He Q, Li S and Qin X: The relationship between red cell distribution width and the risk of Henoch-Schönlein purpura nephritis. Br J Biomed Sci 75: 30-35, 2018.

28. Kim DS, Shin D, Kim TG, Kim SH, Kim DY, Kim SM and Lee MG: Red blood cell distribution width as a useful indicator to predict systemic vasculitis in patients with cutaneous vasculitis. Rheumatol Int 35: 719-725, 2015.

29. Liu Q, Dang AM, Chen BW, Lv NQ, Wang X and Zheng DY: The association of red blood cell distribution width with anemia and inflammation in patients with Takayasu arteritis. Clin Chim Acta 438: 205-209, 2015.

30. Kim HJ, Yoo J, Jung SM, Song JJ, Park YB and Lee SW: Red blood cell distribution width can predict vasculitis activity and poor prognosis in granulomatosis with polyangiitis. Yonsei Med J 59: 294-302, 2018.

31. Aksoy ŞN, Savaş E, Sucu M, Kisacik B, Kul S and Zengin O: Association between red blood cell distribution width and disease activity in patients with Behçet's disease. J Int Med Res 43: 765-773, 2015.

32. Li ZZ, Chen L, Yuan H, Zhou T and Kuang ZM: Relationship between red blood cell distribution width and early-stage renal function damage in patients with essential hypertension. J Hypertens 32: 2450-2455, 2014.

33. Zhang M, Zhang Y, Li C and He L: Association between red blood cell distribution and renal function in patients with untreated type 2 diabetes mellitus. Ren Fail 37: 659-663, 2015.

34. Ujszaszi A, Molnar MZ, Czira ME, Novak M and Mucsi I: Renal function is independently associated with red cell distribution width in kidney transplant recipients: A potential new auxiliary parameter for the clinical evaluation of patients with chronic kidney disease. Br J Haematol 161: 715-725, 2013.

35. Wang W, Liu J, Yang YH, Zhai ZG, Wang C and Wang J: Red cell distribution width is increased in chronic thromboembolic pulmonary hypertension. Clin Respir J 10: 54-60, 2016.

36. Afsar B, Saglam M, Yuceturk C and Agca E: The relationship between red cell distribution width with erythropoietin resistance in iron replete hemodialysis patients. Eur J Intern Med 24: e25-e29, 2013.

This work is licensed under a Creative Commons Attribution-NonCommercial-NoDerivatives 4.0 International (CC BY-NC-ND 4.0) License. 